\title{
Multiview 3D Markerless Human Pose Estimation from OpenPose Skeletons
}

\author{
Maarten Slembrouck ${ }^{1[0000-0002-1822-3881]}$, Hiep Luong ${ }^{1}$, Joeri Gerlo ${ }^{3}$, \\ Kurt Schütte $^{2}$, Dimitri Van Cauwelaert ${ }^{1}$, Dirk De Clercq ${ }^{3}$, Benedicte \\ Vanwanseele $^{2}$, Peter Veelaert ${ }^{1}$, and Wilfried Phillips ${ }^{1}$ \\ 1 TELIN-IPI, Faculty of Engineering and Architecture, Ghent University-imec, \\ Ghent, Belgium (https://ipi.ugent.be) \\ maarten.slembrouck@ugent. be \\ ${ }^{2}$ Human Movement Biomechanics Research Group, Department of Kinesiology, KU \\ Leuven, Leuven, Belgium \\ ${ }^{3}$ Department of Movement and Sport Sciences, Ghent University, Ghent, Belgium
}

\begin{abstract}
Despite the fact that marker-based systems for human motion estimation provide very accurate tracking of the human body joints (at $\mathrm{mm}$ precision), these systems are often intrusive or even impossible to use depending on the circumstances, e.g. markers cannot be put on an athlete during competition. Instrumenting an athlete with the appropriate number of markers requires a lot of time and these markers may fall off during the analysis, which leads to incomplete data and requires new data capturing sessions and hence a waste of time and effort. Therefore, we present a novel multiview video-based markerless system that uses 2D joint detections per view (from OpenPose) to estimate their corresponding 3D positions while tackling the people association problem in the process to allow the tracking of multiple persons at the same time. Our proposed system can perform the tracking in real-time at 20-25 fps. Our results show a standard deviation between 9.6 and $23.7 \mathrm{~mm}$ for the lower body joints based on the raw measurements only. After filtering the data, the standard deviation drops to a range between 6.6 and $21.3 \mathrm{~mm}$. Our proposed solution can be applied to a large number of applications, ranging from sports analysis to virtual classrooms where submillimeter precision is not necessarily required, but where the use of markers is impractical.
\end{abstract}

Keywords: Markerless human motion $\cdot$ joint detection $\cdot$ multiview

\section{Introduction}

Current experiments in the field of human motion analysis are often analysed with marker-based systems such as Qualisys or Vicon. The biggest drawback of these systems is the time needed to instrument a person with reflective markers. Moreover, during the data capturing process markers may fall off, rendering that recording useless. Beside this, such a system with markers cannot be deployed in 


\section{Slembrouck et al.}

numerous applications such as virtual classrooms, athlete analysis during competition and many more.

Recent advances in markerless monocular skeleton detection enable new applications that require semi-accurate tracking of body parts. Such markerless systems provide the solution for the above mentioned drawbacks of markerbased systems. Whereas marker-based systems claim submillimeter accuracy for the markers, markerless systems only obtain an accuracy up to a few centimeters. The reason is that a joint (e.g. an ankle) is not always detected at the anatomically correct position. Depending on the clothing, joints might not even be visible and even humans would have a hard time to locate the exact position of the joints from the videos only.

The changes in planar joint angles are often used for movement analysis, e.g. technical performance in sports or basic clinical gait analysis. For this reason a markerless system has its value despite the fact that it cannot accurately measure rotations along the limbs axis. Markerless systems have been around for a while now. Since the early 2000s, research has been going on to find the location of joints in RGB videos. Most of these approaches relied on shape-fromsilhouettes and tried to match a detailed kinematic model. Positional errors were typically larger than $10 \mathrm{~cm}[6,7,15,16]$. Later advances obtained 51 to 100 $\mathrm{mm}$ positional errors on the joints $[12,13]$. More recently, the shift to monocular pose extraction enabled more flexible camera setups $[8,9]$. However, the reported positional errors of these systems are all typically between 56 and $140 \mathrm{~mm}$. The multiview system that we propose goes one step further. Our system can accurately detect $2 \mathrm{D}$ joints, which enables us to obtain positional errors between 24.2 and $49.2 \mathrm{~mm}$.

Apart from obtaining a better accuracy, we also aim at improved robustness. To obtain this goal, we use the existing 2D pose extractor of OpenPose [3] and triangulate the joint positions in 3D. However, we noticed a number of issues, such as self-occlusion, switching limbs and misdetected joints by the $2 \mathrm{D}$ pose extractor. We handle all three issues in this paper and present a robust system that can be applied in a wide range of applications due to its flexibility in the number of cameras and the scale in which it can be applied. In the results section, we will show promising results that demonstrate this flexibility.

The outline of the paper is as follows. In Section 2 we discuss the pose extraction from monocular video. In Section 3 triangulation is explained for at least two image points from different cameras. We explain how we match different persons in the people association step in Section 4 and in Section 5 the fusion from $2 \mathrm{D}$ to $3 \mathrm{D}$ at a skeleton level is explained. In Section 6 we discuss two use cases where a single person is analysed with 3 and 8 cameras.

\section{Real-time skeleton detection}

Our goals is to have an accurate robust system that runs in real-time, i.e. at a framerate of at least $20 \mathrm{fps}$. OpenPose $[3,4]$ is one of the deep learning algorithms that provides real-time skeleton data. OpenPose is currently the only framework 
to support 25 joint points per person, which makes it very useful to analyse human motion. Our system uses the detected skeletons from OpenPose to reason further about the 3D position of each joint using multiple camera views. Alternative pose extractors were also researched, such as Alpha Pose [10], Cascaded Pyramid Networks [5], Dense Pose [1], PoseFlow [17] and SMPL [2]. However, they only support a limited number of joints to be detected. VNect on the other hand is a close competitor to OpenPose and it also supports for instance the detection of the foot tip, but we were unable to obtain the needed source code to use this framework in our experiments [14]. OpenPose is able to run on an NVidia 1080Ti GPU card at almost $30 \mathrm{fps}$. We employ two graphics cards in parallel and equally distribute the load over both cards. By stitching multiple images together before feeding it to the OpenPose neural network, we are able to process multiple camera images at a rate of 20-25 fps. More convenient GPUs will run the proposed method between 10 and $15 \mathrm{fps}$.

In the next section we will discuss how we estimate $3 \mathrm{D}$ position from a set of $2 \mathrm{D}$ points using least squares triangulation. We need this step for the people association in Section 4 as well as the fusion of skeleton point into their 3D positions, taking into account possible misdetections in 2D in Section 5.

\section{Triangulation}

Traditional cameras observe the $3 \mathrm{D}$ world by light ray projection on a $2 \mathrm{D}$ plane. During this process depth information is lost, which is one of the reasons why it is hard to accurately reconstruct a 3D scene from a single captured image. The use of multiple cameras from multiple viewpoints has proven that $3 \mathrm{D}$ reconstruction is possible by using the content of the image and calibration data of the different cameras [11].

The mathematical conversion from a set of $2 \mathrm{D}$ points from multiple cameras into a $3 \mathrm{D}$ location is often referred to as triangulation. An example of triangulation is shown in Figure 1. The idea is to estimate the position of point $X$ based on the $2 \mathrm{D}$ image positions $p_{i}$ and fixed known camera calibrations (intrinsic and extrinsic). Due to inaccuracies in the camera calibration and the discretization of the image sensor, the lines will rarely intersect. Therefore, we need to apply an approximated model. In the following paragraphs, we will briefly discuss how we perform triangulation using the minimization of least squares of the distances between the point $X$ and the lines defined by the camera position and the pixel location.

For a single joint, we define two vectors: $\boldsymbol{c}_{i}$ which represents the camera position of camera $C_{i}$ and vector $\boldsymbol{a}_{i}$ which is the vector between the unknown $3 \mathrm{D}$ position of the joint $\boldsymbol{x}$ and $\boldsymbol{c}_{i}: \boldsymbol{a}_{i}=\boldsymbol{x}-\boldsymbol{c}_{i}$.

Let the $i$ th line be defined by $\boldsymbol{c}_{i}$ and a unit vector $\boldsymbol{d}_{i}$. Given the principal point $\left(u_{0}^{i}, v_{0}^{i}\right)$ of camera $i$, we can calculate $\boldsymbol{d}_{i}$ as follows:

$$
\widetilde{\boldsymbol{d}}_{t, i}=R_{i}^{T}\left(\begin{array}{c}
m_{i x}\left(p_{i, u}-u_{0}^{i}\right) \\
m_{i y}\left(p_{i, v}-v_{0}^{i}\right) \\
f_{i}
\end{array}\right)
$$


M. Slembrouck et al.

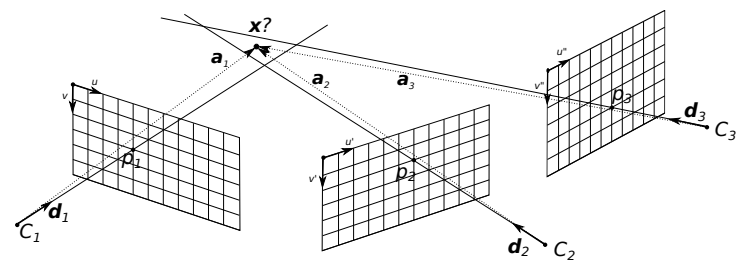

Fig. 1. Triangulation example where we need to estimate the $3 \mathrm{D}$ position of point $X$ based on $2 \mathrm{D}$ points from multiple cameras. Ideally vectors $\boldsymbol{a}_{i}$ and $\boldsymbol{d}_{i}$ would coincide.

$$
\boldsymbol{d}_{i}=\frac{\widetilde{\boldsymbol{d}}_{t, i}}{\left\|\widetilde{\boldsymbol{d}}_{t, i}\right\|}
$$

where $R_{i}$ is the rotation matrix, $f_{i}$ is the focal length $(\mathrm{mm}), m_{i x}$ and $m_{i y}$ represent the horizontal and vertical pixel size on the sensor $(\mathrm{mm})$ and $\left(p_{i, u}, p_{i, v}\right)$ the projected joint position.

The point to line distance is given by:

$$
\left\|\boldsymbol{w}_{i}\right\|=\sqrt{\boldsymbol{w}_{i} \cdot \boldsymbol{w}_{i}} \text { where } \boldsymbol{w}_{i}=\boldsymbol{d}_{i} \times \boldsymbol{a}_{i} .
$$

We now determine the single $3 \mathrm{D}$ point $\boldsymbol{x}$ that minimizes the sum of squared point to line distances $\sum_{i}\left\|\boldsymbol{w}_{i}\right\|^{2}$. This minimum occurs where the gradient is the zero vector $(\mathbf{0})$ :

$$
\nabla\left(\sum_{i}\left\|\boldsymbol{w}_{i}\right\|^{2}\right)=\mathbf{0} .
$$

Expanding the gradient,

$$
\sum_{i}\left(2 \boldsymbol{d}_{i}\left(\boldsymbol{d}_{i} \cdot \boldsymbol{a}_{i}\right)-2\left(\boldsymbol{d}_{i} \cdot \boldsymbol{d}_{i}\right) \boldsymbol{a}_{i}\right)=\mathbf{0}
$$

We found that the coordinates of $\boldsymbol{x}$ satisfy a $3 \times 3$ linear system,

$$
M \boldsymbol{x}=\boldsymbol{b},
$$

where the $k$ th row (a 3 -element row vector) of matrix $M$ is defined as

$$
M_{k}=\sum_{i}\left(d_{i k} \boldsymbol{d}_{i}-\left(\boldsymbol{d}_{i} \cdot \boldsymbol{d}_{i}\right) \boldsymbol{e}_{k}\right)^{T}
$$

with vector $\boldsymbol{e}_{k}$ the respective unit basis vector, and

$$
\boldsymbol{b}=\sum_{i} \boldsymbol{d}_{i}\left(\boldsymbol{c}_{i} \cdot \boldsymbol{d}_{i}\right)-\boldsymbol{c}_{i}\left(\boldsymbol{d}_{i} \cdot \boldsymbol{d}_{i}\right)
$$

In practice $M$ is almost always not singular. However, in rare circumstances, $M$ could be singular. For example, we could have a system with only two cameras facing each other and a joint close close to the line joining the 2 projection centres. Since this situation is highly unlikely, and can be easily avoided, we exclude it. We use Gaussian elimination to find $\boldsymbol{x}$ in Equation 2.

Note that self-occlusion of a joint is automatically solved by not taking into account a viewpoint that did not detect the joint. However, at least two cameras need to observe a joint to report a position. 


\begin{tabular}{c|c} 
camera & \# persons \\
\hline C1 & 3 \\
C2 & 2 \\
C3 & 2 \\
C4 & 3
\end{tabular}

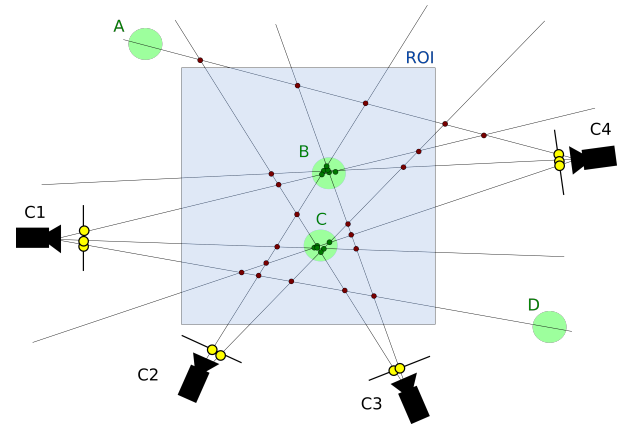

Fig. 2. Clustering of pairwise camera point reconstructions to find different people in the scene. The table indicates the number of detected skeletons in each view. Persons $\mathrm{B}$ and $\mathrm{C}$ are found, Persons $\mathrm{A}$ and $\mathrm{D}$ are located outside the region on interest (ROI).

\section{People association}

Triangulation of a person's joints relies heavily on the assumptions that the $2 \mathrm{D}$ point correspondences in multiple views belong to the same 3D point. However, in case of multiple persons in the area of interest, these correspondences are not so easy to find due to occlusion and possible inaccurate $2 \mathrm{D}$ joint positions. As illustrated in Figure 2.

Let us consider a simple camera network with 4 cameras (Figure 2). For clarity, we have reduced all skeletons to a single point in the figure. Note that each of these points actually represent 25 joints. The number of possible matches between the different $2 \mathrm{D}$ skeletons becomes $(3+1)(2+1)(2+1)(3+1)=144$. We add 1 for each view because none of the skeleton views may correspond to a $3 \mathrm{D}$ skeleton in the area of interest. From the 144 combinations, there are also some combinations that cannot result in a skeleton reconstruction since a minimum of two points from different views is required for triangulation.

There is no need to test all 25 individual joints from the same skeleton combinations. To tackle the people association problem, we calculate pairwise correspondences between skeletons from different viewpoints. We assume that joints from a detected skeleton belong to the same person and the spine is correctly detected which is the case in our applications. Therefore, we only consider the spine of a person, defined by the neck and midhip joint (other joints may be chosen for a different application). Valid combinations are those that correspond to a low point to line distance between the triangulated point and the line defined by its $2 \mathrm{D}$ detection and the camera position for both the neck and the midhip (in the example, two non-parallel lines will always interest. In 3D however, these lines rarely intersect resulting in a reprojection error). In a second phase, the valid matches are clustered based on the 3D distances between the detected spines. 2D skeletons that have been clustered are removed from the search space to avoid that these combinations are again matched with other skeletons later on. Therefore, multiple persons can be calculated from the same set of frames. Additional constraints concerning the region of interest (ROI) may be used to reject persons that are detected in multiple cameras, but are not inside the ROI. 
M. Slembrouck et al.

\section{Fusion of $2 \mathrm{D}$ joint projections into $3 \mathrm{D}$ joints}

The conversion to a $3 \mathrm{D}$ point from multiple $2 \mathrm{D}$ joint projections in different views has been discussed in detail above. However, a number of difficulties needs to be addressed, specifically when it comes to pose estimation.

Triangulation supposes that the detected $2 \mathrm{D}$ points are accurate. A pose extractor, such as OpenPose, provides a confidence score in range 0.0-1.0 for each of the detected joints. Usually the position of joints is rather accurate. However, when the confidence score of joints is low (e.g. below 0.2), we noticed that we better discard these points in the triangulation process. Another issue with the pose extractor of OpenPose is confusion in a sense of the left and right extremities of a person's body. We especially noticed this problem with the legs. In most cases, the left leg is detected on the left side of the person, but sometimes the left leg is confused with the right leg, or both legs are detected inside the same leg depending on the pose of the person of interest. Both issues demand a suitable solution to avoid discrete changes in the spatio-temporal domain. With a limited number of cameras, it is not always clear what the correct solution should be, especially when multiple cameras suffer from left/right confusion at the same time. We need to be careful to swap the limbs in the correct view and not to pose swapping on correct views. In that case the $3 \mathrm{D}$ positions of both legs are correct, with a low point to line distances, but the label left/right might be switched, causing inferior results. Therefore spatio-temporal tracking offers a suitable solution. Figure 3 shows errors that occur frequently in our datasets.
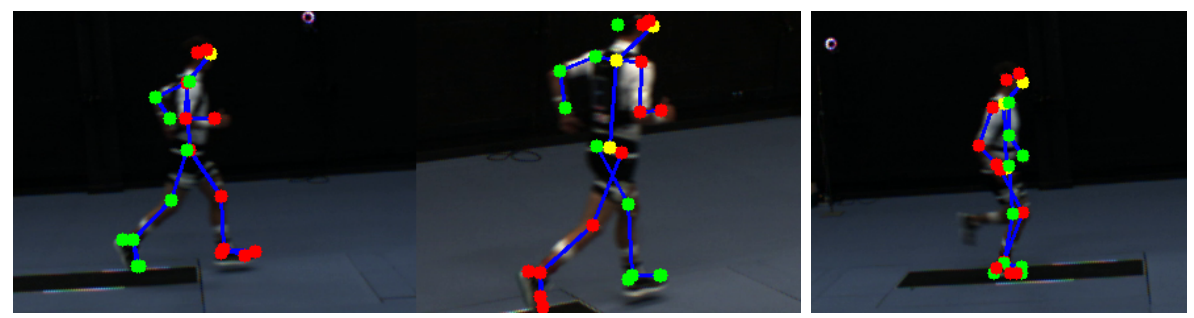

Fig. 3. Misdetected joints causing difficulties in the 3D matching. OpenPose confused between left (red) and right (green) in two different views captured at the same time and both the left and right leg are detected inside the physical right leg, while the physical left leg remains undetected.

\subsection{Handling limb ambiguities}

After reconstructing a skeleton, we calculate the point to line distances between the reconstructed joint position $X_{j}$ and the line defined by the camera position $C_{j}$ and the image location on the image sensor $p_{i j}$ and store them in matrix $D$ which has $n$ rows and $m$ columns where $n$ is the number of cameras and $m$ the number of joints. Only the following are considered in this matrix because they handle switching legs: LHip, LKnee, LAnkle, LHeel, LBigToe, LSmallToe, RHip, RKnee, RAnkle, RHeel, RBigToe and RSmallToe. The same can be done for the arms of a person. We define matrix $D$ as 


$$
D=\left[\begin{array}{ccccc}
\left\|\boldsymbol{w}_{11}\right\|^{2} & \left\|\boldsymbol{w}_{12}\right\|^{2} & \left\|\boldsymbol{w}_{13}\right\|^{2} & \ldots & \left\|\boldsymbol{w}_{1 m}^{2}\right\| \\
\left\|\boldsymbol{w}_{21}\right\|^{2} & \left\|\boldsymbol{w}_{22}\right\|^{2} & \left\|\boldsymbol{w}_{23}\right\|^{2} & \ldots & \left\|\boldsymbol{w}_{2 m}^{2}\right\| \\
\vdots & \vdots & \vdots & \ddots & \vdots \\
\left\|\boldsymbol{w}_{n 1}\right\|^{2} & \left\|\boldsymbol{w}_{n 2}\right\|^{2} & \left\|\boldsymbol{w}_{n 3}\right\|^{2} & \ldots & \left\|\boldsymbol{w}_{n m}^{2}\right\|
\end{array}\right]
$$

where $\left\|\boldsymbol{w}_{i j}\right\|^{2}$ is the squared point to line distance between the $3 \mathrm{D}$ joint position $\boldsymbol{x}_{j}$ and its detected position on camera $j$ (cfr. Equation 1). We choose point to line distances over projection errors because the former takes into account the distance between the camera and the detected point.

\subsection{Minimizing the squared point to line distances in matrix $D$}

Our goal is to remove all limb ambiguities so that we obtain the minimum $\sum_{i} \sum_{j}\left\|\boldsymbol{w}_{i j}\right\|^{2}$. In order to facilitate real-time processing, we limit the combinations. Some are more likely than others e.g. chances are rather small that the limbs of a skeleton have been switched by more than half of the cameras. Therefore, we constrain the search space to reduce the number of limb reassignments. We are satisfied when all values in $D$ are below a certain threshold $T_{m}$ (maximum allowed point to line distance). We also use an additional threshold $T_{u}$ with $T_{m}<T_{u}$ to detect and to cope with extreme cases. Both thresholds are not very sensitive. We found that $T_{m}=100 \mathrm{~mm}$ and $T_{u}=500 \mathrm{~mm}$ correctly fixed the issues with the $2 \mathrm{D}$ skeleton detection.

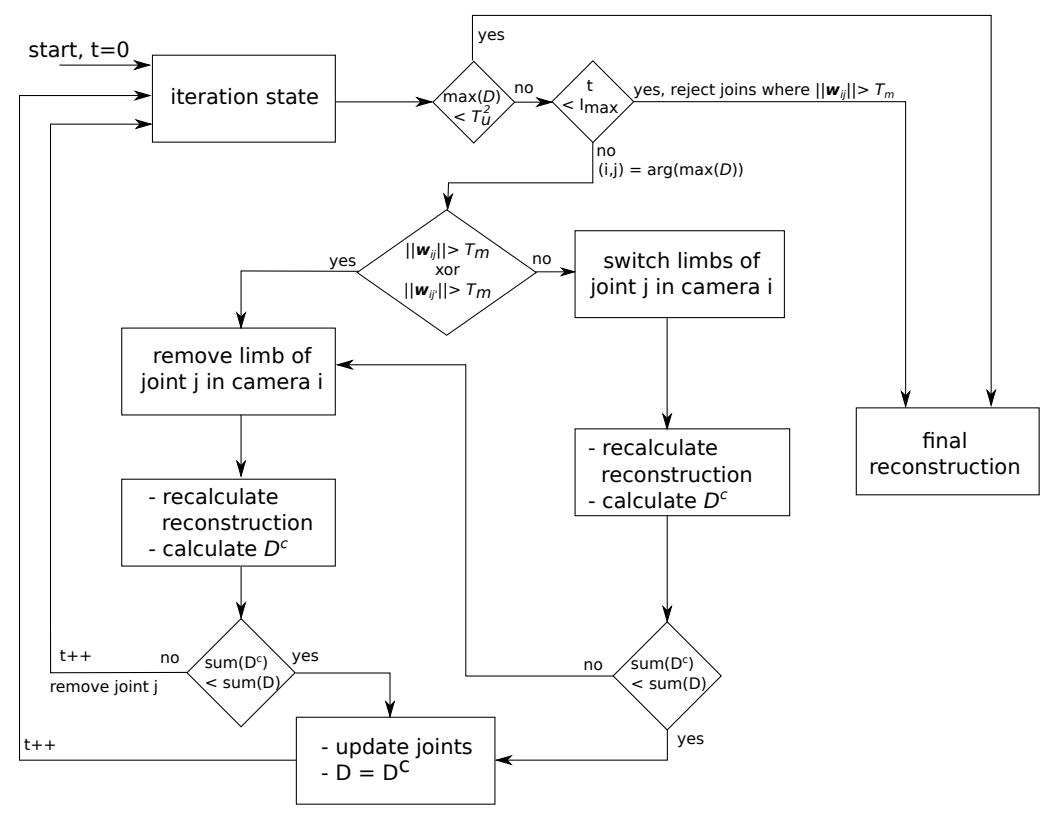

Fig. 4. Flowchart of the proposed algorithm. Joint $j^{\prime}$ represents the opposite joint $j$. 


\section{Slembrouck et al.}

Figure 4 illustrates how the reconstruction is made while coping with switching limbs and misdetected joints. The algorithm starts with the calculation of matrix $D$. We first check if the maximum value in $D$ is lower than $T_{u}^{2}$. If this is the case, we immediately arrive at the final reconstruction. If this is not the case, we verify if the number of iterations $t$ is smaller than $I_{\max }$. If that is the case, we decide not to investigate any longer to maintain the real-time processing time of the algorithm. Depending on the application, we choose to leave out the joints from the final reconstruction to make sure that no faulty positions are returned.

At each iteration the following steps are taken: when only one of the two legs has a point to line distance higher than $T_{m}^{2}$, we are almost certain that both legs are detected inside the same leg, meaning one correct location and one incorrect location. Therefore, we decide to test the point to line distances for the reconstruction without the positions of this leg of that particular camera. If both $x_{i j}$ and $x_{i j^{\prime}}$ are higher than $T_{m}^{2}$, we switch the limbs first. If that does not lead to a better reconstruction, we ignore the limb as well. In all cases, the reconstruction is only accepted, when the sum of the errors of the new candidate joints $D^{C}$ decreases: $\operatorname{sum}\left(D^{C}\right)<\operatorname{sum}(D)$.

\section{Experiments}

Two experiments were conducted on two different locations which both were recorded with vision-based cameras and infra-red cameras. The marker-based camera systems (Qualisys and Vicon) have a theoretical submillimeter precision for the marker positions. However, we should keep in mind that due to marker/soft tissue movement it is unlikely that submillimeter precision is reached for the calculation of joint center positions.

The first dataset was recorded at the Sports Science Laboratory Jacques Rogge (SSL-JR) at Ghent University. The vision-based system consisted out of seven 4.5 MP cameras (Manta G-046C, AVT, Stadtroda, Germany). The person of interest ran in a straight line, always in the same direction at different speeds ranging from 2.1 to $5.1 \mathrm{~m} / \mathrm{s}$ (Figure 5). The camera images are captured synchronously by two computers at $67 \mathrm{~Hz}$. The running length that can be captured is around 11 meters. The infra-red based motion capture system consisted out of ten 1.3 MP cameras (Oqus3+, Qualisys AB, Gteborg, Sweden) operating at a frame rate of $250 \mathrm{~Hz}$. The cameras were fixed to the lab walls, uniformly distributed to measure $4 \mathrm{~m}$ of the running length, with a distance to the center of the volume ranging from 3.5 to $7 \mathrm{~m}$. In total 88 Passive IR-reflective 12 mm-sized spherical markers were attached to the subject body and used for full body modeling in Visual 3D software (C-Motion Inc., Germantown, USA). Joint center coordinates of the ankles, knees and hips were exported for comparison. The wand calibration of the setup showed a standard deviation on measured distances of $0.4 \mathrm{~mm}$.

The second dataset was recorded in Leuven, Belgium. Only three 4.5 MP cameras (Manta G-046C, AVT, Stadtroda, Germany) were used operating at a frame rate of $50 \mathrm{~Hz}$. The cameras were located closer to the person of interest in 

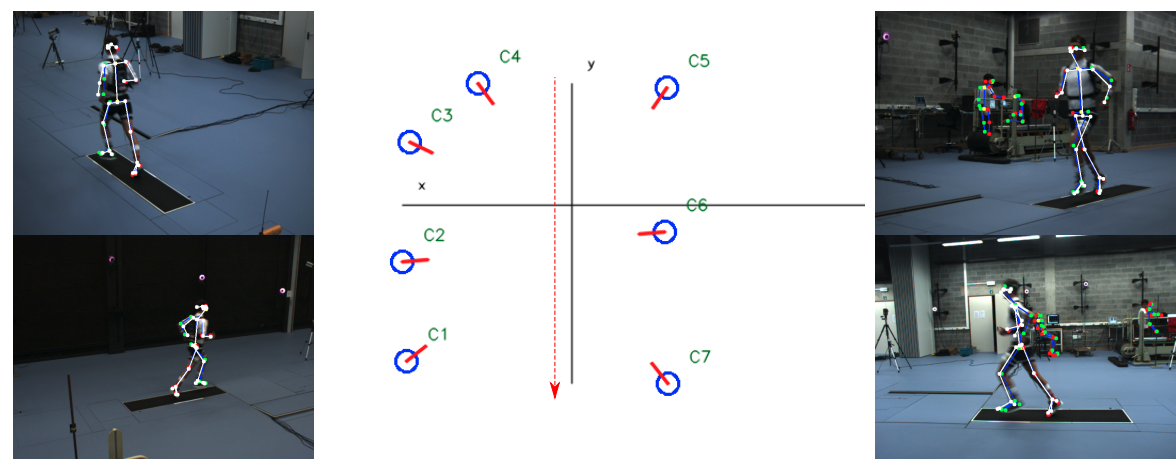

Fig. 5. Camera setup in the Sport Science Lab Jacques Rogge in Ghent on the left (SSL-JR dataset). The red arrow indicated the running direction. On the right we show two camera images with detected 3D skeleton (white) on top. The yellow and cyan lines represent the people association.

comparison with the first dataset because the measuring volume was only $3 \times 3 \times$ 2 meters. The person in this dataset is executing stationary movements such as squats and clocks (Figure 6). A fixed ten camera Vicon system (Vicon MX T20, VICON Motion Systems Ltd., Oxford, UK) supplemented with three additional portable Vicon Vero cameras (Vicon Vero v1.3, VICON Motion Systems Ltd., Oxford, UK) were used. All cameras were sampled at $100 \mathrm{~Hz}$ and utilizing a measurement error of $1 \mathrm{~mm}$. In addition, ground reaction forces were collected using two AMTI OR 6 Series force plates sampled at $1000 \mathrm{~Hz}$ (Optima, Advanced Mechanical Technology, Inc., Watertown, USA). These force plates were used to determine initial ground contact during the side cut maneuver and check the execution of the clock for the Vicon system. A single researcher placed 39 retro-reflective markers on the participant using palpation to identify the correct attachment site. Markers were placed as shown in Figure 6 on the trunk, pelvis and both legs and feet, in order to collect kinematic data for the trunk, hip, knee and ankle.
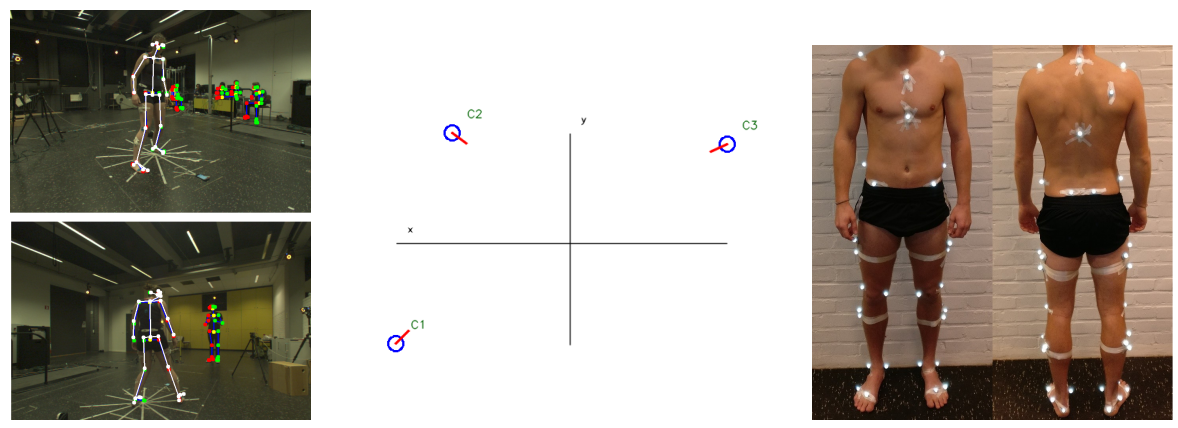

Fig. 6. 3-camera setup in Leuven positioned approximately $2.5 \mathrm{~m}$ from the person. 


\section{Slembrouck et al.}

First, we take a look at the graphical representation of the individual results, after which we will evaluate results averaged over all sequences and the spread that can be found in these measurements. We filtered the raw measurements in the spatio-temporal domain from the frame by frame results with a Hanning window of length 7 . Such operation slightly improves the results. Figure 7 shows a typical graph produced by the proposed system. We see that the proposed system follows the marker-based positions rather accurately.

Table 1 shows the accuracy averaged per dataset, while Figure 8 shows the distribution of these numbers. We may conclude that spatio-temporal filtering improves the results by decreasing the standard deviation and average positional error between 1 and $3 \mathrm{~mm}$. For the second dataset we notice an offset in positional errors for a number of joints. The limited number of cameras is most likely the cause of this. Also the cameras in this setup are not entirely evenly distributed around the person of interest. However, the experiments show that even with a limited number of cameras, the proposed method performs well. The offsets between the marker-based and proposed system, bares little significance because the standard deviation is small we reliably detects position changes of the different joints even in case of self-occlusion.
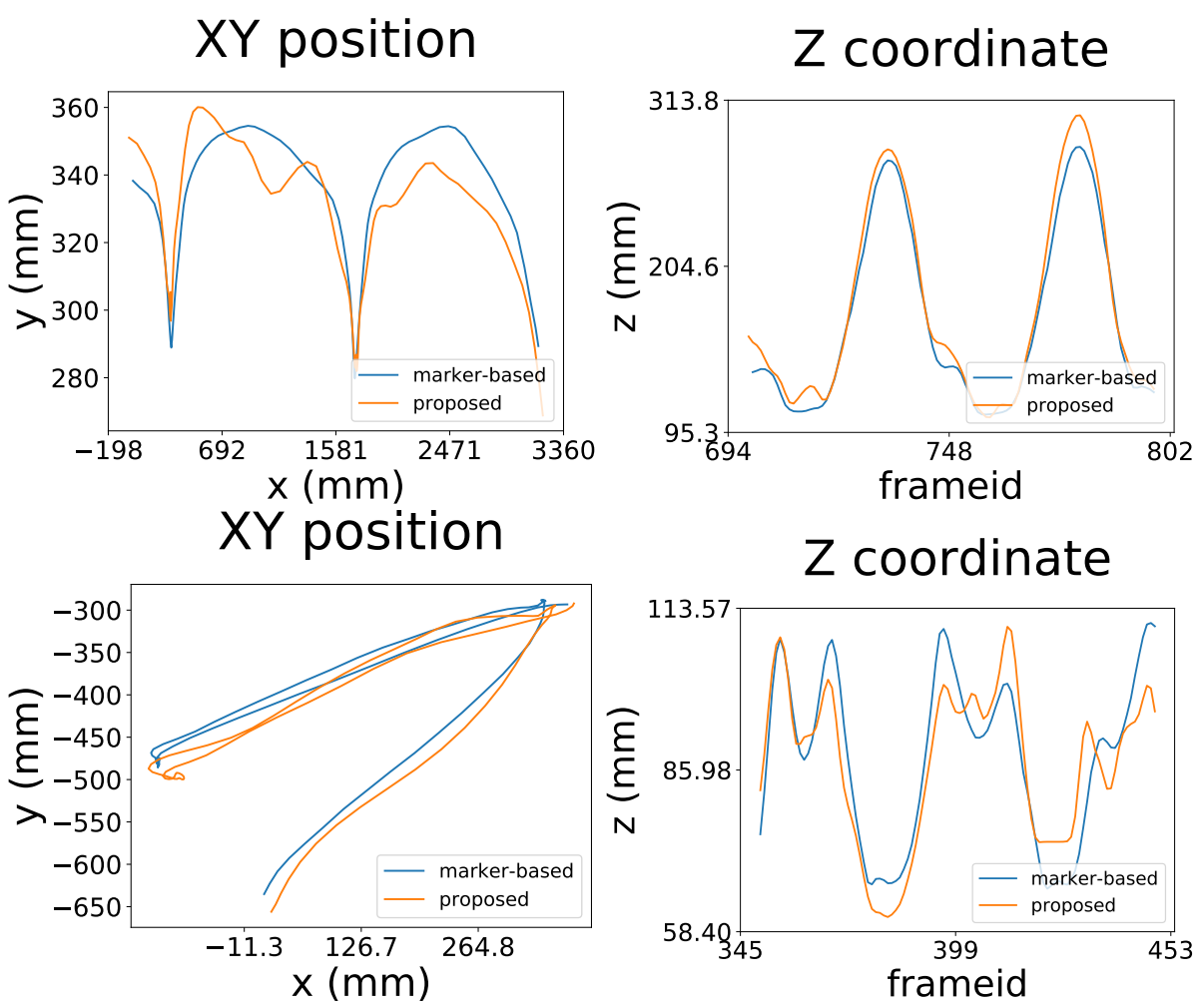

Fig. 7. Typical result of the positions of an ankle joint (top row: SSL-JR dataset, bottom row IPLAY-Leuven dataset). Note the different scales in each of the graphs. 

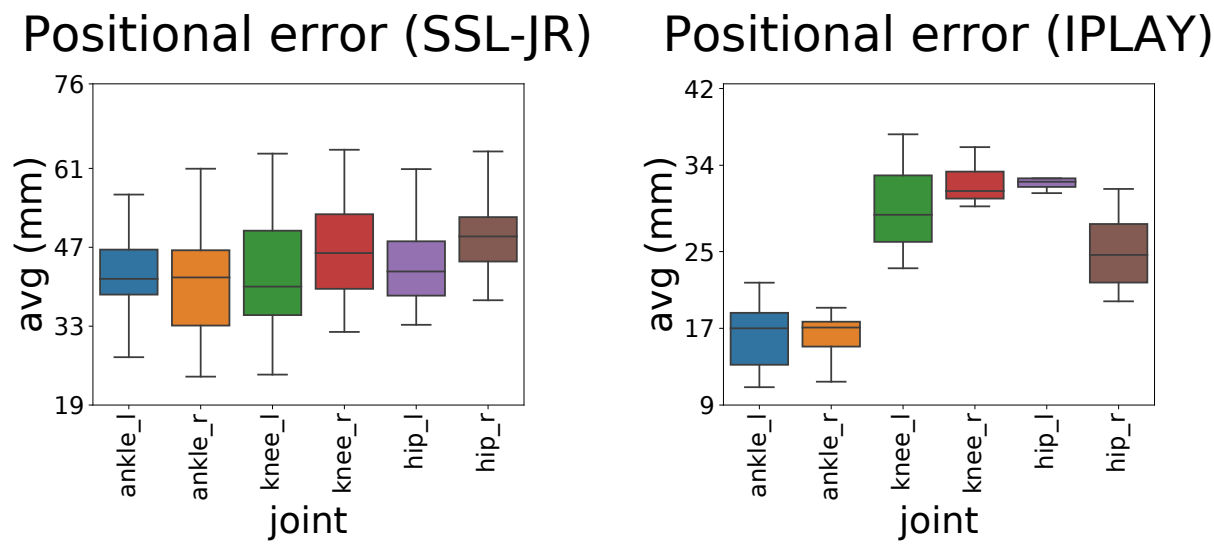

Fig. 8. Average positional error between the marker-based positions and the estimated $3 \mathrm{D}$ position for all 33 sequences (SSL-JR) and 9 sequences (IPLAY).

\begin{tabular}{|c|c||c|c||c|r|}
\hline & \multicolumn{1}{|c||}{} & \multicolumn{2}{c||}{ Unfiltered } & \multicolumn{2}{c|}{ Filtered } \\
& joint & avg & stddev & avg & stddev \\
\hline S & ankle_l & 42.1 & 23.7 & 40.6 & 21.3 \\
S & ankle_r & 41.2 & 21.7 & 38.8 & 19.4 \\
L & knee_l & 42.2 & 20.8 & 40.4 & 19.1 \\
J & knee_r & 46.3 & 19.9 & 44.8 & 18.3 \\
R & hip_l & 44.4 & 18.8 & 41.6 & 15.5 \\
& hip_r & 50.7 & 15.2 & 49.2 & 12.5 \\
\hline I & ankle_l & 19.4 & 11.9 & 18.7 & 8.2 \\
P & ankle_r & 16.7 & 9.6 & 15.9 & 6.6 \\
L & knee_l & 30.3 & 10.6 & 29.8 & 7.9 \\
A & knee_r & 32.6 & 12.4 & 32.1 & 10.0 \\
Y & hip_l & 34.2 & 15.7 & 33.5 & 10.9 \\
& hip_r & 26.1 & 14.7 & 25.1 & 11.0 \\
\hline
\end{tabular}

Table 1. Positional errors and standard deviation averaged over 33 sequences (SSL-JR) and 9 sequences (IPLAY-Leuven dataset). All measurements are in $\mathrm{mm}$.

\section{Conclusion}

In this paper we presented a fast and reliable way to convert 2D OpenPose skeleton detections from multiple camera views into 3D skeletons. Our proposed method copes with misdetected joints and switching limbs to extract reliable 3D tracking data for 25 joints of the human body. During our experiments we found that the positional error for the lower limbs are between 15.9 and $49.2 \mathrm{~mm}$ and the standard deviation between 6.6 and $21.3 \mathrm{~mm}$. We compared our system to marker-based systems, which claim submillimeter accuracy. The reported accuracy is not as precise as marker-based systems, but much more flexible and can be used in applications which are satisfied with an accuracy of a few millimeter such as entertainment applications, macro body analysis, virtual classrooms... 
M. Slembrouck et al.

\section{Acknowledgement}

This work was supported by the imec.ICON IPLAY project and a joint cooperation between the Faculty of Engineering and the Faculty of Medicine and Health Sciences at Ghent University. We would like to acknowledge Maxim Steinmeyer and Maarten Van Dyck for their assistance with participant recruitment, data collection and labeling of the IPLAY-Leuven dataset.

\section{References}

1. Alp Güler, R., Neverova, N., Kokkinos, I.: Densepose: Dense human pose estimation in the wild. In: CVPR. pp. 7297-7306 (2018)

2. Bogo, F., Kanazawa, A., Lassner, C., Gehler, P., Romero, J., Black, M.J.: Keep it SMPL: Automatic estimation of 3D human pose and shape from a single image. In: ECCV. Springer (Oct 2016)

3. Cao, Z., Hidalgo, G., Simon, T., Wei, S.E., Sheikh, Y.: OpenPose: realtime multiperson 2D pose estimation using Part Affinity Fields. In: arXiv (2018)

4. Cao, Z., Simon, T., Wei, S.E., Sheikh, Y.: Realtime multi-person 2 d pose estimation using part affinity fields. In: CVPR. pp. 7291-7299 (2017)

5. Chen, Y., Wang, Z., Peng, Y., Zhang, Z., Yu, G., Sun, J.: Cascaded pyramid network for multi-person pose estimation. In: CVPR (June 2018)

6. Cheung, G.K.M., Baker, S., Hodgins, J., Kanade, T.: Markerless human motion transfer. In: 3DPVT. pp. 373-378 (Sep 2004)

7. Cheung, K., Baker, S., Kanade, T.: Shape-from-silhouette of articulated objects and its use for human body kinematics estimation and motion capture. In: CVPR. IEEE (2003)

8. Du, Y., Wong, Y., Liu, Y., Han, F., Gui, Y., Wang, Z., Kankanhalli, M., Geng, W.: Marker-less 3d human motion capture with monocular image sequence and height-maps. In: ECCV. pp. 20-36. Springer (2016)

9. Elhayek, A., Kovalenko, O., Murthy, P., Malik, J., Stricker, D.: Fully automatic multi-person human motion capture for vr applications. In: EuroVR (2018)

10. Fang, H.S., Xie, S., Tai, Y.W., Lu, C.: RMPE: Regional multi-person pose estimation. In: ICCV (2017)

11. Hartley, R.I., Sturm, P.: Triangulation. In: Hlaváč, V., Šára, R. (eds.) Computer Analysis of Images and Patterns. pp. 190-197. Springer, Berlin, Heidelberg (1995)

12. Hofmann, M., Gavrila, D.M.: Multi-view 3d human pose estimation combining single-frame recovery, temporal integration and model adaptation. In: CVPR. pp. 2214-2221 (June 2009)

13. Huo, F., Hendriks, E., Paclik, P., Oomes, A.H.: Markerless human motion capture and pose recognition. In: 2009 10th Workshop on Image Analysis for Multimedia Interactive Services. pp. 13-16. IEEE (2009)

14. Mehta, D., Sridhar, S., Sotnychenko, O., Rhodin, H., Shafiei, M., Seidel, H.P., Xu, W., Casas, D., Theobalt, C.: Vnect: Real-time 3d human pose estimation with a single rgb camera. ACM Transactions on Graphics 36(4), 44 (2017)

15. Rosenhahn, B., Kersting, U.G., Smith, A.W., Gurney, J.K., Brox, T., Klette, R.: A system for marker-less human motion estimation. In: Pattern Recognition. pp. 230-237. Springer, Berlin, Heidelberg (2005)

16. Saboune, J., Charpillet, F.: Markerless human motion capture for gait analysis. arXiv (2005)

17. Xiu, Y., Li, J., Wang, H., Fang, Y., Lu, C.: Pose Flow: Efficient online pose tracking. In: BMVC (2018) 